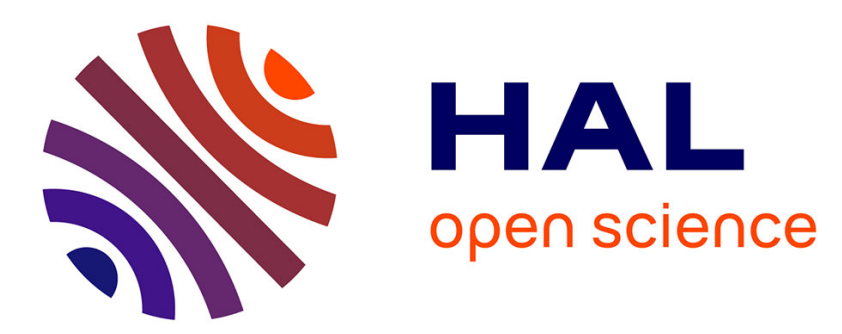

\title{
Weighted Sum Rate Maximization of Correlated MISO Broadcast Channels under Linear Precoding: A Large System Analysis
}

Sebastian Wagner, Dirk Slock

\section{To cite this version:}

Sebastian Wagner, Dirk Slock. Weighted Sum Rate Maximization of Correlated MISO Broadcast Channels under Linear Precoding: A Large System Analysis. 12th IEEE International Workshop on Signal Processing Advances in Wireless Communications, Jun 2011, San Francisco, United States. pp.411 - 415, 10.1109/SPAWC.2011.5990441 . hal-00675524

\section{HAL Id: hal-00675524 \\ https://hal.science/hal-00675524}

Submitted on 1 Mar 2012

HAL is a multi-disciplinary open access archive for the deposit and dissemination of scientific research documents, whether they are published or not. The documents may come from teaching and research institutions in France or abroad, or from public or private research centers.
L'archive ouverte pluridisciplinaire HAL, est destinée au dépôt et à la diffusion de documents scientifiques de niveau recherche, publiés ou non, émanant des établissements d'enseignement et de recherche français ou étrangers, des laboratoires publics ou privés. 


\title{
Weighted Sum Rate Maximization of Correlated MISO Broadcast Channels under Linear Precoding: A Large System Analysis
}

\author{
Sebastian Wagner, Student Member, IEEE, and Dirk T. M. Slock, Fellow, IEEE
}

\begin{abstract}
In this paper, we study the iterative weighted sum rate (WSR) maximizing linear precoder algorithm proposed by Christensen et al. in large correlated MISO broadcast channels. We propose a novel approximation of the signal-to-interference plus noise ratio (SINR) at every iteration. Moreover, for independent and identical distributed channels the approximation of the WSR maximizing precoder is of closed form and has the well known regularized zero-forcing (RZF) structure. Simulation results show that under correlated channels, the novel SINR approximation is more accurate than previously proposed estimates based on the RZF precoder.
\end{abstract}

Index Terms-random matrix theory, beamforming, weighted sum rate maximization.

\section{INTRODUCTION}

W E consider the multiple-input single-output (MISO) broadcast channel (BC) with linear precoding at the transmitter. In this case, the base station (BS) is endowed with $M$ antennas, whereas the $K$ users have single-antenna receivers. The precoding matrix that maximizes (local optimum) the weighted sum rate (WSR) is obtained from an iterative algorithm [1]. Due to its iterative nature, this optimal precoder does not lend itself to analytic optimization problems that might give insightful results. Simple precoder such as zero-forcing (ZF) or regularized ZF (RZF) [2] are much more attractive in this regard. Therefore, it is important to understand the connection between the optimal iterative precoder and the suboptimal closed-form precoders. It has been shown in [3] that iterative optimal precoder has the RZF structure, only when the channel is symmetric. Furthermore, it has been claimed in [4] that for large dimensions $M, K \rightarrow \infty$ with finite ratio $\beta \triangleq M / K$ and independent and identical distributed (i.i.d.) channels, the optimal linear precoder has the RZF structure as well.

In this contribution, we carry out a large system analysis of the iterative precoder proposed in [1]. Hereby, we extend the work in [5], [6] by deriving a novel approximation of the signal-to-interference plus noise ratio (SINR) at every iteration for large $M, K$. For correlated channels, this new approximation outperforms the one proposed in [5], [6] and is identical to the RZF approximation for i.i.d. channels [4], [7].

The analysis leads to the following results, (i) under peruser channel correlation, the SINR approximation is iterative

S. Wagner is with ST-ERICSSON, Sophia-Antipolis

S. Wagner and D.T.M. Slock are with EURECOM, SophiaAntipolis, 06904, Route des Crêtes, B.P. 193, France email: $\{$ sebastian.wagner, dirk.slock\}@eurecom.fr. as well and is more accurate than the estimate obtained by a simplified RZF approach and (ii) for i.i.d. channels, the precoder is of closed-form and has the RZF structure as proposed in [4]

Simulations show, that the proposed SINR approximation offers a good performance and is close to optimal.

Notation: In the following, boldface lower-case and uppercase characters denote vectors and matrices, respectively. The operators $(\cdot)^{\mathrm{H}}, \operatorname{tr}(\cdot)$ and $E[\cdot]$ denote conjugate transpose, trace and expectation, respectively. The $N \times N$ identity matrix is denoted $\mathbf{I}_{N}$ and $\log (\cdot)$ is the natural logarithm.

\section{SySTEM MODEL}

Consider a transmitter endowed with $M$ antennas communicating with $K$ single-antenna receivers, $k=1,2, \ldots, K$. Under narrow-band transmission, the received signal $y_{k}$ reads

$$
y_{k}=\mathbf{h}_{k}^{\mathrm{H}} \mathbf{x}+n_{k},
$$

where $\mathbf{h}_{k}^{\mathrm{H}} \in \mathbb{C}^{1 \times M}$ is the channel vector of user $k, \mathbf{x} \in \mathbb{C}^{M}$ is the transmit vector and the $n_{k}$ are independent complex Gaussian noise terms with zero mean and variance $\sigma^{2}$.

We assume that $\mathbf{h}_{k}^{\mathrm{H}}$ is correlated as $E\left[\mathbf{h}_{k} \mathbf{h}_{k}^{\mathrm{H}}\right]=\boldsymbol{\Theta}_{k}$ and thus can be written in the form

$$
\mathbf{h}_{k}=\sqrt{M} \Theta_{k}^{1 / 2} \mathbf{z}_{k},
$$

where $\mathbf{z}_{k}$ has i.i.d. complex entries of zero mean and variance $1 / M$ and the $\boldsymbol{\Theta}_{k}^{1 / 2}$ is any Hermitian square-root of $\boldsymbol{\Theta}_{k}$. The correlation matrix $\boldsymbol{\Theta}_{k}$ is nonnegative Hermitian and of uniformly bounded spectral norm w.r.t. to $M$.

Prior to transmission, the user symbols $s_{k} \sim \mathcal{N C}(0,1)$ are linearly precoded and form the transmit signal

$$
\mathbf{x}=\sum_{k=1}^{K} \mathbf{g}_{k} s_{k},
$$

where $\mathbf{g}_{k} \in \mathbb{C}^{M}$ is the precoding vector of user $k$. The transmitter is subject to an average power constraint

$$
E\left[\mathbf{x}^{\mathrm{H}} \mathbf{x}\right]=\operatorname{tr} \mathbf{G} \mathbf{G}^{\mathrm{H}} \leq P,
$$

where $\mathbf{G}=\left[\mathbf{g}_{1}, \mathbf{g}_{2}, \ldots, \mathbf{g}_{K}\right] \in \mathbb{C}^{M \times K}$ is the precoding matrix and $P$ is the total available transmit power.

Under optimal single-user decoding, the achievable rate of user $k$ is given by

$$
R_{k}=\log \left(1+\gamma_{k}\right)
$$


where $\gamma_{k}$ is the SINR of user $k$ and reads

$$
\gamma_{k}=\frac{\left|\mathbf{h}_{k}^{\mathrm{H}} \mathbf{g}_{k}\right|^{2}}{\sum_{i \neq k}^{K} \mathbf{h}_{k}^{\mathrm{H}} \mathbf{g}_{i} \mathbf{g}_{i}^{\mathrm{H}} \mathbf{h}_{k}+\sigma^{2}} .
$$

The precoding vectors are chosen to maximize the weighted sum rate of all users and the optimization problem takes the form

$$
\begin{gathered}
\mathbf{G}^{\star}=\underset{\mathbf{G}}{\arg \min } \sum_{k=1}^{K}-u_{k} R_{k} \\
\text { s.t. } \quad \operatorname{tr} \mathbf{G G}^{\mathrm{H}} \leq P,
\end{gathered}
$$

where $u_{k} \geq 0$ is the weight of user $k$. The optimization problem in (7) is hard to solve directly, since it is highly nonconvex in the precoding matrix $\mathbf{G}$. To solve the problem in (7), consider the virtual linear receive filters $a_{k} \in \mathbb{C}$. The error variance $e_{k}$ after the linear receive filtering reads

$$
e_{k}=E\left[\left(a_{k} y_{k}-s_{k}\right)\left(a_{k} y_{k}-s_{k}\right)^{\mathrm{H}}\right] \text {. }
$$

By introducing additional weighting scalars $w_{k}$, the cost function (7) can be modified and an equivalent optimization problem can be formulated as [1]

$$
\begin{gathered}
\left\{\mathbf{G}^{\star}, a_{k}^{\star}, w_{k}^{\star}\right\}=\underset{\mathbf{G}, a_{k}, w_{k}}{\arg \min }\left\{u_{k}\left[w_{k} e_{k}-\log \left(u_{k}^{-1} w_{k}\right)-1\right]\right\} \\
\text { s.t. } \operatorname{tr} \mathbf{G} \mathbf{G}^{\mathrm{H}} \leq P .
\end{gathered}
$$

Denote $\rho \triangleq P / \sigma^{2}$ the signal-to-noise ratio (SNR). From (9), the optimal filters can be derived easily and read

$$
\begin{aligned}
a_{k}^{\star} & =\sigma \mathbf{g}_{k}^{\mathrm{H}} \mathbf{h}_{k}\left(\sigma^{2}+\mathbf{h}_{k}^{\mathrm{H}} \mathbf{G} \mathbf{G}^{\mathrm{H}} \mathbf{h}_{k}\right)^{-1} \\
e_{k}^{\star} & =\left(1+\gamma_{k}\right)^{-1} \\
w_{k}^{\star} & =u_{k}\left(e_{k}^{\star}\right)^{-1} \\
\tilde{\mathbf{G}}^{\star} & =\left(\mathbf{H}^{\mathrm{H}} \mathbf{D H}+\frac{\operatorname{tr} \mathbf{D}}{\rho} \mathbf{I}_{M}\right)^{-1} \mathbf{H}^{\mathrm{H}} \mathbf{A}^{\mathrm{H}} \mathbf{W},
\end{aligned}
$$

where $\mathbf{G}^{\star}=\xi \tilde{\mathbf{G}}^{\star}$ with $\xi=\sqrt{P / \operatorname{tr} \tilde{\mathbf{G}}^{\star} \tilde{\mathbf{G}}^{\star \mathrm{H}}}$. Also we defined $\mathbf{W} \triangleq \operatorname{diag}\left(w_{1}^{\star}, \ldots, w_{K}^{\star}\right), \mathbf{A} \triangleq \operatorname{diag}\left(a_{1}^{\star}, \ldots, a_{K}^{\star}\right), \mathbf{D} \triangleq$ $\mathbf{A}^{\mathrm{H}} \mathbf{W A}$ and $\mathbf{H} \triangleq\left[\mathbf{h}_{1}, \ldots, \mathbf{h}_{K}\right]^{\mathrm{H}} \in \mathbb{C}^{K \times M}$ is the compound channel. For notational convenience, we drop the superscript $\star$ in the sequel.

In this paper, we base our analysis on the iterative algorithm called WSRBF-WMMSE2 proposed in [1]. Here, the precoding matrix is initialized with the transmit matched filter (MF). Subsequently $a_{k}$ and $w_{k}$ are computed, which then constitute the new precoder $\mathbf{G}$. This process is repeated until convergence to a local optimum.

\section{LARGE SYSTEM ANALYSIS}

In this section, under a simplifying assumption, we derive an approximation $\overline{\gamma_{k}}$ of the SINR $\gamma_{k}$ in (6) such that $\gamma_{k}$ $\overline{\gamma_{k}} \stackrel{M \rightarrow \infty}{\longrightarrow} 0$, almost surely. This approximation is referred to as deterministic equivalent.

At every iteration of the WSRBF-WMMSE2 algorithm, we calculate $\bar{\gamma}_{k}$, a deterministic equivalent of the SINR $\gamma_{k}$. Since the algorithm is iterative, $\bar{\gamma}_{k}^{(j)}$ at iteration $j$ depends in a non-trivial way on the channel $\mathbf{H}$, making it difficult if not impossible to calculate. Therefore, we study the following precoder $\overline{\mathbf{G}}^{(j)}=\xi^{(j)} \tilde{\mathbf{G}}^{(j)}$ at iteration $j$, where

$$
\tilde{\overline{\mathbf{G}}}^{(j)}=\left(\mathbf{H}^{\mathrm{H}} \overline{\mathbf{D}}^{(j)} \mathbf{H}+\frac{\operatorname{tr} \overline{\mathbf{D}}^{(j)}}{\rho} \mathbf{I}_{M}\right)^{-1} \mathbf{H}^{\mathbf{H}} \overline{\mathbf{A}}^{\mathrm{H},(j)} \overline{\mathbf{W}}^{(j)},
$$

where $\xi^{(j)}=\sqrt{P / \operatorname{tr} \tilde{\overline{\mathbf{G}}}^{(j)} \tilde{\overline{\mathbf{G}}}^{\mathrm{H},(j)}}$ and $\overline{\mathbf{D}}^{(j)}=$ $\operatorname{diag}\left(\bar{d}_{1}^{(j)}, \ldots, \bar{d}_{K}^{(j)}\right), \quad \overline{\mathbf{A}}^{\mathbf{H},(j)}=\operatorname{diag}\left(\bar{a}_{1}^{(j)}, \ldots, \bar{a}_{K}^{(j)}\right) \quad$ and $\overline{\mathbf{W}}^{(j)}=\operatorname{diag}\left(\bar{w}_{1}^{(j)}, \ldots, \bar{w}_{K}^{(j)}\right)$ such that $d_{k}^{(j)}-\bar{d}_{k}^{(j)} \stackrel{M \rightarrow \infty}{\longrightarrow} 0$, $a_{k}^{(j)}-\bar{a}_{k}^{(j)} \stackrel{M \rightarrow \infty}{\longrightarrow} 0$ and $w_{k}^{(j)}-\bar{w}_{k}^{(j)} \stackrel{M \rightarrow \infty}{\longrightarrow} 0$ almost surely, respectively.

\section{A. Deterministic Equivalent of the SINR for the MF}

For the large system analysis, it is unimportant how we initialize the filters $\mathbf{g}_{k}$, because after the first iteration, the structure of the precoder is given by (14). As in the original algorithm in [1], we choose the transmit MF, i.e., $\mathbf{g}_{k}^{(0)}=\mathbf{h}_{k}$. A deterministic equivalent $\bar{\gamma}_{k}^{\mathrm{MF}}$ of the SINR $\gamma_{k}^{\mathrm{MF}}$ under matched filter (MF) precoding given by (6), such that $\gamma_{k}^{\mathrm{MF}}-\bar{\gamma}_{k}^{\mathrm{MF}} \stackrel{M \rightarrow \infty}{\longrightarrow}$ 0 , almost surely, is given in the following theorem. We require $\bar{\gamma}_{k}^{\mathrm{MF}}$ to compute $\bar{\gamma}_{k}^{(1)}$ in Theorem 2.

Theorem 1: Let $\gamma_{k}^{\mathrm{MF}}$ be the SINR of user $k$ under MF precoding, i.e., $\mathbf{G}=\frac{\xi}{M} \mathbf{H}^{\mathrm{H}}$. Then, $\gamma_{k}^{\mathrm{MF}}-\bar{\gamma}_{k}^{\mathrm{MF}} \stackrel{M \rightarrow \infty}{\longrightarrow} 0$, almost surely, where

$$
\bar{\gamma}_{k}^{\mathrm{MF}}=\frac{1}{\frac{1}{\beta \rho}+\frac{1}{M^{2}} \sum_{i \neq k}^{K} \operatorname{tr} \boldsymbol{\Theta}_{k} \boldsymbol{\Theta}_{i}} .
$$

Proof: The normalization parameter is $\xi=\sqrt{\frac{P}{\frac{1}{M^{2}} \operatorname{tr} \mathbf{H}^{\mathrm{H}} \mathbf{H}}}$ and thus with (10) we have

$$
\bar{\xi}=\sqrt{\frac{P}{\frac{1}{M^{2}} \sum_{k=1}^{K} \operatorname{tr} \Theta_{k}}}=\sqrt{\beta P} .
$$

Denote $P_{S, k} \triangleq\left|\mathbf{g}_{k}^{\mathrm{H}} \mathbf{h}_{k}\right|^{2}$ the signal power of user $k$. Applying [8, Lemma 2.7] we have $\frac{1}{M} \mathbf{h}_{k}^{\mathrm{H}} \mathbf{h}_{k}-1 \stackrel{M \rightarrow \infty}{\longrightarrow} 0$ and hence

$$
\bar{P}_{S, k}=\bar{\xi}^{2}=\beta P \text {. }
$$

The interference is $\frac{\xi^{2}}{M} \mathbf{z}_{k}^{\mathrm{H}} \boldsymbol{\Theta}_{k}^{1 / 2} \mathbf{H}_{[k]}^{\mathrm{H}} \mathbf{H}_{[k]} \boldsymbol{\Theta}_{k}^{1 / 2} \mathbf{z}_{k}$, where $\mathbf{H}_{[k]}=$ $\left[\mathbf{h}_{1}, \ldots, \mathbf{h}_{k-1}, \mathbf{h}_{k+1}, \ldots, \mathbf{h}_{K}\right]^{\mathrm{H}}$. Now we apply again [8, Lemma 2.7] since $\frac{1}{M} \Theta_{k}^{1 / 2} \mathbf{H}_{[k]}^{\mathrm{H}} \mathbf{H}_{[k]} \Theta_{k}^{1 / 2}$ has uniformly bounded spectral norm w.r.t. $M$ almost surely, and obtain

$$
\frac{1}{M} \mathbf{z}_{k}^{\mathrm{H}} \boldsymbol{\Theta}_{k}^{1 / 2} \mathbf{H}_{[k]}^{\mathrm{H}} \mathbf{H}_{[k]} \boldsymbol{\Theta}_{k}^{1 / 2} \mathbf{z}_{k}-\frac{1}{M^{2}} \sum_{i \neq k}^{K} \operatorname{tr} \boldsymbol{\Theta}_{k} \boldsymbol{\Theta}_{i} \stackrel{M \rightarrow \infty}{\longrightarrow} 0
$$

almost surely. Substituting the terms in (6) by their respective deterministic equivalents yields (15), which completes the proof.

\section{B. Deterministic equivalent of the SINR of proposed precoder for correlated channels}

For the precoder (14), a deterministic equivalent of the SINR is provided in the following theorem. 
Theorem 2: Let $\gamma_{k}$ be the SINR of user $k$ with the precoder defined in (14). Then, a deterministic equivalent $\bar{\gamma}_{k}^{(j)}$ at iteration $j>0$ and under MF initialization $\bar{G}^{(0)}=\frac{\xi^{(0)}}{M} \mathbf{H}^{\mathrm{H}}$, is given by

$$
\gamma_{k}^{(j)}-\bar{\gamma}_{k}^{(j)} \stackrel{M \rightarrow \infty}{\longrightarrow} 0
$$

almost surely, where $\bar{\gamma}_{k}^{(j)}$ is given by

$$
\bar{\gamma}_{k}^{(j)}=\frac{\bar{w}_{k}^{(j)}\left(\bar{m}_{k}^{(j)}\right)^{2}}{\bar{\Upsilon}_{k}^{(j)}+\bar{d}_{k}^{(j)} \frac{\bar{\Psi}^{(j)}}{\rho}\left(1+\bar{m}_{k}^{(j)}\right)^{2}},
$$

where

$$
\begin{aligned}
& \bar{m}_{k}^{(j)}=\frac{1}{M} \operatorname{tr} \overline{\boldsymbol{\Theta}}_{k}^{(j)} \mathbf{V}, \\
& \bar{\Psi}^{(j)}=\frac{1}{M} \sum_{i=1}^{K} \frac{\bar{w}_{i}^{(j)} e_{i}^{\prime}}{\left(1+e_{i}\right)^{2}} \\
& \bar{\Upsilon}_{k}^{(j)}=\frac{1}{M} \sum_{i=1}^{K} \frac{\bar{w}_{i}^{(j)} e_{i, k}^{\prime}}{\left(1+e_{i}\right)^{2}},
\end{aligned}
$$

and $\bar{a}_{k}^{(j)}, \bar{w}_{k}^{(j)}$ and $\bar{d}_{k}^{(j)}$ are given by

$$
\begin{aligned}
\bar{a}_{k}^{(j)} & =\frac{\sigma}{\sqrt{\bar{P}_{S, k}^{(j-1)}}} \frac{\bar{\gamma}_{k}^{(j-1)}}{1+\bar{\gamma}_{k}^{(j-1)}} \\
\sqrt{\bar{P}_{S, k}^{(j-1)}} & =\frac{1}{\bar{a}_{k}^{(j-1)}} \sqrt{\frac{P}{\bar{\Psi}^{(j-1)}}} \frac{\bar{m}_{k}^{(j-1)}}{1+\bar{m}_{k}^{(j-1)}} \\
\bar{w}_{k}^{(j)} & =u_{k}\left(1+\bar{\gamma}_{k}^{(j-1)}\right) \\
\bar{d}_{k}^{(j)} & =\bar{w}_{k}^{(j)} \bar{a}_{k}^{2,(j)}
\end{aligned}
$$

Denoting $\mathbf{V} \triangleq\left(\mathbf{F}+\alpha \mathbf{I}_{M}\right)^{-1}$, three systems of $K$ coupled equations have to be solved. First, the $e_{1}, \ldots, e_{K}$ form the unique positive solutions of

$$
\begin{aligned}
e_{i} & =\frac{1}{M} \operatorname{tr} \boldsymbol{\Theta}_{i} \mathbf{V}, \\
\mathbf{F} & =\frac{1}{M} \sum_{j=1}^{K} \frac{\boldsymbol{\Theta}_{j}}{1+e_{j}} .
\end{aligned}
$$

Secondly, the $e_{1}^{\prime}, \ldots, e_{K}^{\prime}$ form the unique positive solutions of

$$
\begin{aligned}
e_{i}^{\prime} & =\frac{1}{M} \operatorname{tr} \boldsymbol{\Theta}_{i} \mathbf{V}^{2}\left(\mathbf{F}^{\prime}+\mathbf{I}_{M}\right), \\
\mathbf{F}^{\prime} & =\frac{1}{M} \sum_{j=1}^{K} \frac{\boldsymbol{\Theta}_{j} e_{j}^{\prime}}{\left(1+e_{j}\right)^{2}} .
\end{aligned}
$$

Finally, the $e_{1, k}^{\prime}, \ldots, e_{K, k}^{\prime}$ are the unique positive solutions of

$$
\begin{aligned}
e_{i, k}^{\prime} & =\frac{1}{M} \operatorname{tr} \boldsymbol{\Theta}_{i} \mathbf{V}^{2}\left(\mathbf{F}_{k}^{\prime}+\boldsymbol{\Theta}_{k}\right), \\
\mathbf{F}_{k}^{\prime} & =\frac{1}{M} \sum_{j=1}^{K} \frac{\boldsymbol{\Theta}_{j} e_{j, k}^{\prime}}{\left(1+e_{j}\right)^{2}} .
\end{aligned}
$$

For $j=1, \bar{\gamma}_{k}^{(0)}=\bar{\gamma}_{k}^{\mathrm{MF}}$, given by Theorem 1 and $\bar{P}_{S, k}^{(0)}=\beta P$, cf. (17).
Proof: For $j \geq 1$, define $\boldsymbol{\Gamma}^{(j)} \triangleq \frac{1}{M} \mathbf{H}^{\mathrm{H}} \overline{\mathbf{D}}^{(j)} \mathbf{H}+\bar{\alpha}^{(j)} \mathbf{I}_{M}$, with $\bar{\alpha}^{(j)} \triangleq \frac{\operatorname{tr} \overline{\mathbf{D}}^{(j)}}{M \rho}$ the precoder at the end of iteration $j$ is given by

$$
\overline{\mathbf{G}}^{(j)}=\frac{\xi^{(j)}}{M}\left(\boldsymbol{\Gamma}^{(j)}\right)^{-1} \mathbf{H}^{\mathrm{H}} \overline{\mathbf{A}}^{\mathbf{H},(j)} \overline{\mathbf{W}}^{(j)},
$$

where $\xi^{(j)}$ is

$$
\begin{aligned}
\xi^{(j)} & =\sqrt{\frac{P}{\frac{1}{M^{2}} \operatorname{tr}\left(\Gamma^{(j)}\right)^{-2} \mathbf{H}^{\mathrm{H}} \overline{\mathbf{A}}^{\mathbf{H},(j)} \overline{\mathbf{W}}^{2,(j)} \overline{\mathbf{A}}^{(j)} \mathbf{H}}} \\
& \triangleq \sqrt{\frac{P}{\Psi^{(j)}}} .
\end{aligned}
$$

The derivation of the deterministic equivalents of the normalization term $\xi^{(j)}$, the signal power $\left|\overline{\mathbf{g}}_{k}^{\mathrm{H},(j)} \mathbf{h}_{k}\right|^{2}$ and the interference power $\sum_{i \neq k}^{K} \mathbf{h}_{k}^{\mathrm{H}} \overline{\mathbf{g}}_{i}^{(j)} \overline{\mathbf{g}}_{i}^{\mathrm{H},(j)} \mathbf{h}_{k}$ can be made equivalent to [5], [6] by replacing $\overline{\boldsymbol{\Theta}}_{k}^{(j)} \triangleq \bar{d}_{k}^{(j)} \boldsymbol{\Theta}_{k}$ and the user powers by the MMSE weights, i.e., $p_{k}=\bar{w}_{k}^{(j)}$. This will be shown in the following.

a) Power normalization: The term $\Psi^{(j)}$ can be written as

$$
\begin{aligned}
\Psi^{(j)} & =\frac{1}{M^{2}} \operatorname{tr}\left(\boldsymbol{\Gamma}^{(j)}\right)^{-2} \mathbf{H}^{\mathrm{H}} \overline{\mathbf{A}}^{\mathrm{H},(j)} \overline{\mathbf{W}}^{2,(j)} \overline{\mathbf{A}}^{(j)} \mathbf{H} \\
& =\frac{1}{M} \sum_{k=1}^{K} \bar{w}_{k}^{(j)} \bar{d}_{k}^{(j)} \mathbf{z}_{k}^{\mathrm{H}} \boldsymbol{\Theta}_{k}^{1 / 2}\left(\boldsymbol{\Gamma}^{(j)}\right)^{-2} \mathbf{\Theta}_{k}^{1 / 2} \mathbf{z}_{k} \\
& =\frac{1}{M} \sum_{k=1}^{K} \bar{w}_{k}^{(j)} \mathbf{z}_{k}^{\mathrm{H}} \overline{\boldsymbol{\Theta}}_{k}^{1 / 2,(j)}\left(\boldsymbol{\Gamma}^{(j)}\right)^{-2} \overline{\boldsymbol{\Theta}}_{k}^{1 / 2,(j)} \mathbf{z}_{k}
\end{aligned}
$$

Similar to [5], [6], a deterministic equivalent $\bar{\Psi}^{(j)}$ such that $\Psi^{(j)}-\bar{\Psi}^{(j)} \stackrel{M \rightarrow \infty}{\longrightarrow} 0$, almost surely, is given by

$$
\begin{aligned}
\bar{\Psi}^{(j)} & =\frac{1}{M} \sum_{k=1}^{K} \bar{w}_{k}^{(j)} \frac{\frac{1}{M} \operatorname{tr} \overline{\boldsymbol{\Theta}}_{k}^{(j)}\left(\boldsymbol{\Gamma}^{(j)}\right)^{-2}}{\left(1+\frac{1}{M} \operatorname{tr} \overline{\boldsymbol{\Theta}}_{k}^{(j)}\left(\boldsymbol{\Gamma}^{(j)}\right)^{-1}\right)^{2}} \\
& =\frac{1}{M} \sum_{k=1}^{K} \bar{w}_{k}^{(j)} \frac{\bar{m}_{k}^{\prime,(j)}}{\left(1+\bar{m}_{k}^{(j)}\right)^{2}}=\frac{1}{M} \sum_{k=1}^{K} \frac{\bar{w}_{k}^{(j)} e_{k}^{\prime}}{\left(1+e_{k}\right)^{2}},
\end{aligned}
$$

where we denote $\bar{m}_{k}^{(j)} \triangleq \frac{1}{M} \operatorname{tr} \overline{\boldsymbol{\Theta}}_{k}^{(j)}\left(\boldsymbol{\Gamma}^{(j)}\right)^{-1}$ and $\bar{m}_{k}^{\prime,(j)}$ is the derivative w.r.t. $z$ at $z=-\bar{\alpha}^{(j)}$.

b) Signal power: The square-root of the signal power $P_{S, k}^{(j)} \triangleq\left|\overline{\mathbf{g}}_{k}^{\mathrm{H},(j)} \mathbf{h}_{k}\right|^{2}$ is

$$
\begin{aligned}
\sqrt{P_{S, k}^{(j)}} & =\xi^{(j)} \bar{a}_{k}^{(j)} \bar{w}_{k}^{(j)} \mathbf{z}_{k}^{\mathrm{H}} \boldsymbol{\Theta}_{k}^{1 / 2}\left(\boldsymbol{\Gamma}^{(j)}\right)^{-1} \boldsymbol{\Theta}_{k}^{1 / 2} \mathbf{z}_{k} \\
& =\frac{\xi^{(j)}}{\bar{a}_{k}^{(j)}} \mathbf{z}_{k}^{\mathrm{H}} \overline{\boldsymbol{\Theta}}_{k}^{1 / 2,(j)}\left(\boldsymbol{\Gamma}^{(j)}\right)^{-1} \overline{\boldsymbol{\Theta}}_{k}^{1 / 2,(j)} \mathbf{z}_{k} .
\end{aligned}
$$

Again, following [5], [6], a deterministic equivalent $\sqrt{\bar{P}_{S, k}^{(j)}}$ of (42) such that $\sqrt{P_{S, k}^{(j)}}-\sqrt{\bar{P}_{S, k}^{(j)}} \stackrel{M \rightarrow \infty}{\longrightarrow} 0$, almost surely, is given by

$$
\sqrt{\bar{P}_{S, k}^{(j)}}=\frac{\bar{\xi}^{(j)}}{\bar{a}_{k}^{(j)}} \frac{\bar{m}_{k}^{(j)}}{1+\bar{m}_{k}^{(j)}},
$$

where $\bar{\xi}^{(j)}=\sqrt{P / \bar{\Psi}^{(j)}}$. 
c) Interference power: The interference power can be written as

$$
\begin{aligned}
& \sum_{i \neq k}^{K} \mathbf{h}_{k}^{\mathrm{H}} \overline{\mathbf{g}}_{i}^{(j)} \overline{\mathbf{g}}_{i}^{\mathrm{H},(j)} \mathbf{h}_{k} \\
& =\frac{\xi^{2,(j)}}{M^{2}} \mathbf{h}_{k}^{\mathrm{H}}\left(\boldsymbol{\Gamma}^{(j)}\right)^{-1} \sum_{i \neq k}^{K} \bar{a}_{i}^{2,(j)} \bar{w}_{i}^{2,(j)} \mathbf{h}_{i} \mathbf{h}_{i}^{\mathrm{H}}\left(\boldsymbol{\Gamma}^{(j)}\right)^{-1} \mathbf{h}_{k} \\
& =\frac{\xi^{2,(j)}}{\bar{d}_{k}^{(j)}} \mathbf{z}_{k}^{\mathrm{H}} \overline{\mathbf{\Theta}}_{k}^{1 / 2,(j)}\left(\boldsymbol{\Gamma}^{(j)}\right)^{-1} \\
& \times \sum_{i \neq k}^{K} \bar{w}_{i}^{(j)} \overline{\mathbf{\Theta}}_{i}^{1 / 2,(j)} \mathbf{z}_{i} \mathbf{z}_{i}^{\mathrm{H}} \overline{\mathbf{\Theta}}_{i}^{1 / 2,(j)}\left(\boldsymbol{\Gamma}^{(j)}\right)^{-1} \overline{\mathbf{\Theta}}_{k}^{1 / 2, j} \mathbf{z}_{k} .
\end{aligned}
$$

The term in (46) is equivalent to the one in [5], [6] replacing $p_{k}$ by $\bar{w}_{k}^{(j)}$. Thus we have

$$
\sum_{i \neq k}^{K} \mathbf{h}_{k}^{\mathrm{H}} \overline{\mathbf{g}}_{i}^{(j)} \overline{\mathbf{g}}_{i}^{\mathrm{H},(j)} \mathbf{h}_{k}-\frac{\bar{\xi}^{2,(j)} \bar{\Upsilon}_{k}^{(j)}}{\bar{d}_{k}^{(j)}\left(1+\bar{m}_{k}^{(j)}\right)^{2}} \stackrel{M \rightarrow \infty}{\longrightarrow} 0,
$$

almost surely, where $\bar{\Upsilon}_{k}^{(j)}$ is given by (23), which completes the proof.

\section{Uncorrelated Channels: $\Theta_{k}=\mathbf{I}_{M}$}

If the channel is uncorrelated, the precoder in (14) is of closed form and independent of iteration $j$.

Proposition 1: For the precoder (14) and for $\boldsymbol{\Theta}_{k}=\mathbf{I}_{M}$, we have

$$
\overline{\mathbf{G}}=\xi\left(\mathbf{H}^{\mathrm{H}} \mathbf{U} \mathbf{H}+\frac{\operatorname{tr} \mathbf{U}}{\rho} \mathbf{I}_{M}\right)^{-1} \mathbf{H}^{\mathrm{H}},
$$

where $\xi=\sqrt{P / \operatorname{tr} \overline{\mathbf{G}} \overline{\mathbf{G}}^{\mathbf{H}}}$ and $\mathbf{U}=\operatorname{diag}\left(u_{1}, u_{2}, \ldots, u_{K}\right)$.

Proof: From Theorem 2, observe that both $\bar{a}_{k}^{(j)}$ and $\bar{w}_{k}^{(j)} / u_{k}$ are identical for all users and $\overline{\mathbf{D}}^{(j)}=c \mathbf{U}$ where $c=\bar{a}^{2,(j)} \bar{w}_{k}^{(j)} / u_{k}$. Therefore,

$$
\begin{aligned}
\tilde{\overline{\mathbf{G}}}^{(j)} & =c \xi\left(\mathbf{H}^{\mathrm{H}} \mathbf{U} \mathbf{H}+\frac{\operatorname{tr} \mathbf{U}}{\rho} \mathbf{I}_{M}\right)^{-1} \mathbf{H}^{\mathrm{H}} \\
\xi^{2} & =\frac{c^{2} P}{\operatorname{tr}\left(\mathbf{H}^{\mathrm{H}} \mathbf{U} \mathbf{H}+\frac{\operatorname{tr} \mathbf{U}}{\rho} \mathbf{I}_{M}\right)^{-2} \mathbf{H}^{\mathrm{H}} \mathbf{H}},
\end{aligned}
$$

which completes the proof.

Corollary 1: [5], [6] Let $\boldsymbol{\Theta}=\mathbf{I}_{M}$. Under the assumptions of Theorem 2 the deterministic equivalent of the SINR is of closed form an reads

$$
\bar{\gamma}_{k}^{(j)} \triangleq \bar{\gamma}=\frac{1}{2}[\rho(\beta-1)+\chi-1],
$$

where $\chi$ is given by

$$
\chi=\sqrt{(\beta-1)^{2} \rho^{2}+2(1+\beta) \rho+1} .
$$

\section{NumERICAL RESUlTS}

The Monte-Carlo (MC) simulations are carried out over independent Rayleigh block-fading channels $\mathbf{z}_{k}$. We use the WSRBF-WMMSE2 algorithm in [1] with 10 iterations and MF initialization and compare it to the large system approximation in Theorem 2. In all simulations, the approximated SINR in

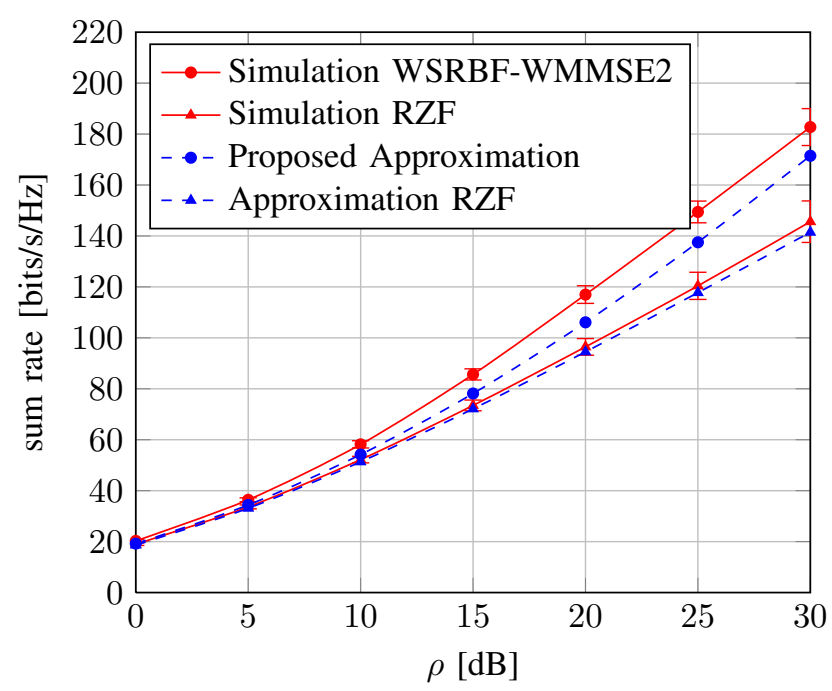

Fig. 1. Sum rate vs. SNR with $M=K=30, \Theta_{k} \neq \mathbf{I}_{M}$, error bars indicating the standard deviation.

Theorem 2 at iteration $j=3$ is computed and an approximated sum rate $\bar{R}_{\text {sum }}=\sum_{k=1}^{K} \log \left(1+\bar{\gamma}_{k}^{(3)}\right)$ is obtained.

The channel correlation matrix is modeled as [9]

$$
\left[\boldsymbol{\Theta}_{k}\right]_{i j}=\frac{1}{\theta_{k, \max }-\theta_{k, \min }} \int_{\theta_{k, \min }}^{\theta_{k, \max }} e^{\mathbf{j} \frac{2 \pi}{\lambda} d_{i j} \cos (\theta)} d \theta,
$$

where $\mathbf{j} \triangleq \sqrt{-1}, \lambda$ denotes the signal wavelength and $d_{i j}$ is the distance between antenna $i$ and $j$. As a simple example we choose the range of azimuth angle $\theta_{k}$ of user $k$ as $\theta_{k, \min }=$ $-\pi$ and $\theta_{k, \max }=\varphi_{k}-\pi$, where $\varphi_{k}=2 \pi k / K$ (uniform user distribution). The transmitter is endowed with a uniform linear array (ULA) of antennas. We assume that $d_{i j}$ is independent of $M$ so that the spectral norm of $\boldsymbol{\Theta}_{k}$ remains bounded as $M$ grows large.

In Figure 1 we compare the optimal iterative linear precoders to the closed-form RZF precoder, in both MC simulations and their respective approximations under the correlation model (53) (indicated $\Theta_{k} \neq \mathbf{I}_{M}$ ). It can be observed that (i) WSRBF-WMMSE2 is superior to RZF and (ii) the approximation for RZF is more accurate than the proposed approximation for the WSRBF-WMMSE2. The first observation is expected, since the RZF is suboptimal. The second observation is explained by the fact that, for RZF it has been proved [6] that the approximation of the SINR is almost surely exact as $M \rightarrow \infty$. Still, the proposed approximation outperforms the RZF approximation and is not too far from optimal.

Moreover, other simulations suggest, that to be more precise, the approximation in Theorem 2 requires larger dimensions than the RZF equivalent.

Figure 2 compares the WSRBF-WMMSE2 precoder and its approximation for correlated channels $\left(\boldsymbol{\Theta}_{k} \neq \mathbf{I}_{M}\right)$ and i.i.d. channels $\left(\boldsymbol{\Theta}_{k}=\mathbf{I}_{M}\right)$. In [4], it has been claimed that for $\boldsymbol{\Theta}_{k}=\mathbf{I}_{M}$ the approximation of the SINR of RZF in Corollary 1 is almost surly optimal as $M \rightarrow \infty$. It can be observed that for i.i.d. channels the approximation in Corollary 1 is accurate for low SNR, but very imprecise at high SNR. This observation suggests that at least significantly higher dimen- 


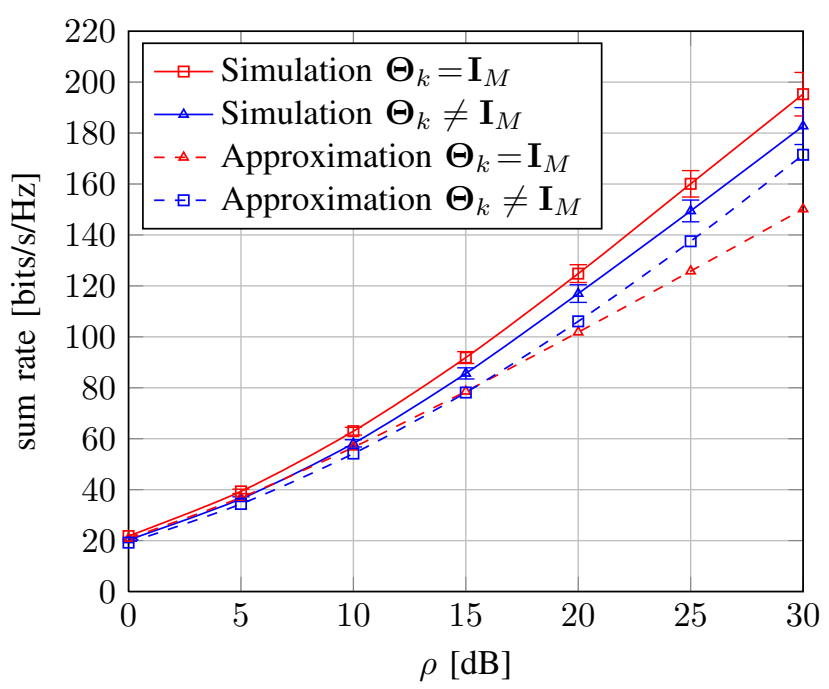

Fig. 2. WSRBF-WMMSE2, sum rate vs. SNR with $M=K=30$, error bars indicating the standard deviation.

sions are required to close the gap between approximation and simulation at high SNR. The reason is, that the regularization term in the inverse of (14) is going to zero for $\rho \rightarrow \infty$ and the maximum eigenvalue of the inverse is fluctuating significantly leading to large standard deviations in the simulations as well as to less accurate approximations. As Figure 2 suggests, this effect is diminished when the channel is correlated resulting in an increased accuracy of the approximation for high SNR.

\section{CONCLUSION}

This paper investigated the behavior of the optimal linear precoder in large correlated MISO broadcast channels. We derived a novel iterative approximation of the SINR and showed that it outperforms the closed-form RZF precoder. The novel approximation is independent of a particular channel realization and thus allows for less complex performance evaluation.

\section{REFERENCES}

[1] S. Christensen, R. Agarwal, and J. M. Cioffi, "Weighted Sum-Rate Maximization using Weighted MMSE for MIMO-BC Beamforming Design," IEEE Trans. Wireless Commun., vol. 7, no. 12, pp. 4792-4799, Dec. 2008.

[2] C. B. Peel, B. M. Hochwald, and A. L. Swindlehurst, "A VectorPerturbation Technique for Near-Capacity Multiantenna Multiuser Communication-Part I: Channel Inversion and Regularization,” IEEE Trans. Commun., vol. 53, no. 1, pp. 195-202, Jan. 2005.

[3] A. Wiesel, Y. C. Eldar, and S. Shamai, "Linear Precoding via Conic Optimization for Fixed MIMO Receivers," IEEE Trans. Signal Process., vol. 54, no. 1, pp. 161-176, Jan. 2006.

[4] R. Zakhour and S. V. Hanly, "Base station cooperation on the downlink: Large system analysis," IEEE Trans. Inf. Theory, Jun. 2010. [Online]. Available: http://arxiv.org/abs/1006.3360

[5] S. Wagner, R. Couillet, M. Debbah, and D. T. M. Slock, "Large System Analysis of Linear Precoding in MISO Broadcast Channels with Limited Feedback," IEEE Trans. Inf. Theory, submitted for publication. [Online]. Available: http://arxiv.org/abs/0906.3682

[6] — "Deterministic Equivalent for the SINR of Regularized Zero-forcing Precoding in Correlated MISO Broadcast Channels with Imperfect CSIT," in Proc. IEEE International Conference on Communications (ICC'11), Kyoto, Japan, Jun. submitted for publication.
[7] V. K. Nguyen and J. S. Evans, "Multiuser Transmit Beamforming via Regularized Channel Inversion: A Large System Analysis," in Proc. IEEE Global Communications Conference (GC'08), New Orleans, LO, Dec. 2008, pp. $1-4$.

[8] Z. D. Bai and J. W. Silverstein, "No Eigenvalues Outside the Support of the Limiting Spectral Distribution of Large Dimensional Sample Covariance Matrices," Annals of Probability, vol. 26, no. 1, pp. 316-345, Jan. 1998.

[9] W. C. Jakes and D. C. Cox, Microwave Mobile Communications. Hoboken, NJ: Wiley-IEEE Press, 1994. 